\title{
Immunomodulatory activity of andrographolide on macrophage activation and specific antibody response
}

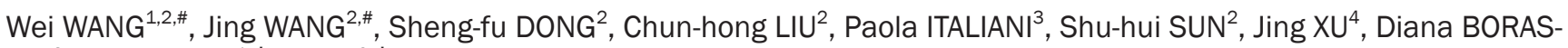
$\mathrm{CHI}^{3}$, Shi-ping $\mathrm{MA}^{1, *}$, Di QU ${ }^{2, *}$

${ }^{1}$ Department of Pharmacology of Chinese Materia Medica, China Pharmaceutical University, Nanjing 210038, China; ${ }^{2}$ Key Laboratory of Medical Molecular Virology of Ministries of Education and Health, Institute of Medical Microbiology and Institutes of Biomedical Sciences, Shanghai Medical College of Fudan University, Shanghai 200032, China; ${ }^{3}$ Laboratory of Cytokines, Unit of Immunobiology, Institute of Biomedical Technologies, National Research Council, Pisa 56124, Italy; ${ }^{4}$ Beijing Institute of Biological Products, Beijing 100024, China

\begin{abstract}
Aim: To investigate the immunomodulatory effects of andrographolide on both innate and adaptive immune responses. Methods: Andrographolide (10 $\mathrm{\mu g} / \mathrm{mL}$ in vitro or $1 \mathrm{mg} / \mathrm{kg}$ in vivo) was used to modulate LPS-induced classical activated (M1) or IL-4induced alternative activated (M2) macrophages in vitro and humor immune response to HBsAg in vivo. Cytokine gene expression profile (M1 vs M2) was measured by real-time PCR, IL-12/IL-10 level was detected by ELISA, and surface antigen expression was evaluated by flow cytometry, whereas phosphorylation level of ERK $1 / 2$ and AKT was determined by Western blot. The level of anti-HBs antibodies in HBsAg immunized mice was detected by ELISA, and the number of HBsAg specific IL-4-producing splenocyte was enumerated by ELISPOT.

Results: Andrographolide treatment in vitro attenuated either LPS or IL-4 induced macrophage activation, inhibited both M1 and M2 cytokines expression and decreased IL-12/IL-10 ratio (the ratio of M1/M2 polarization). Andrographolide down-regulated the expression of mannose receptor (CD206) in IL-4 induced macrophages and major histocompability complex/costimulatory molecules (MHC I, CD40, CD80, CD86) in LPS-induced macrophages. Correspondingly, anti-HBs antibody production and the number of IL-4-producing splenocytes were reduced by in vivo administration of andrographolide. Reduced phosphorylation levels of ERK1/2 and AKT were observed in macrophages treated with andrographolide.

Conclusion: Andrographolide can modulate the innate and adaptive immune responses by regulating macrophage phenotypic polarization and Ag-specific antibody production. MAPK and PI3K signaling pathways may participate in the mechanisms of andrographolide regulating macrophage activation and polarization.
\end{abstract}

Keywords: macrophages; cytokines; antibodies; immunomodulator; andrographolide

Acta Pharmacologica Sinica (2010) 31: 191-201; doi: 10.1038/aps.2009.205

\section{Introduction}

Andrographis paniculata is an herb used in Chinese traditional medicine as recorded in Chinese Pharmacopoeia ${ }^{[1]}$, and a common remedy for anti-infection treatment ${ }^{[2]}$. Andrographolide, a bicyclic diterpenoid lactone, 3-[2-[decahydro-6-hydroxy-5(hydroxylmethyl)-5,8a-dimethyl-2-methylene-1-napthalenyl] ethylidene]-dihydro-4-hydroxy-2(3H)-furanone, is the major

\footnotetext{
\# These two authors contributed equally to this work.

* To whom correspondence should be addressed.

E-mail dqu@shmu.edu.cn (Di QU) spma@cpu.edu.cn (Shi-ping MA)

Received 2009-06-29 Accepted 2009-12-24
}

active constituent and has various pharmacological effects, including anti-inflammation ${ }^{[3]}$, anti-tumor ${ }^{[4]}$, antidiabetic ${ }^{[5]}$ and cardioprotective activities ${ }^{[6]}$. In clinic, andrographolide has being applied as an anti-inflammatory remedy for upper respiratory tract infection and bacterial dysentery. Andrographolide was reported to have immunoregulatory activities. In tumor-bearing mice it enhanced natural killer cell activity ${ }^{[7]}$, increased secretion of IL-2 and IFN- $\gamma$ by T cells and thereby inhibited the tumor growth ${ }^{[8]}$. In autoimmune encephalomyelitis mice it interfered with maturation of dendritic cells ${ }^{[9]}$, induced antigen-specific tolerance and thus prevented detrimental autoimmune responses ${ }^{[10]}$. It suggests that androgra- 
pholide can have different effects in different immune disease models, playing a role as a modulator of altered immune responses rather than a sole immunostimulatory or immunosuppressive agent. Considering that macrophages are widely distributed immune cells that play an indispensable role in homeostasis and defense, we investigated the immunomodulatory effect of andrographolide on the alteration of macrophage phenotype and function.

Macrophages can be activated and phenotypically polarized by different stimuli and microenvironment ${ }^{[11]}$. In general, activated and polarized macrophages can be broadly classified into two main groups: classically activated inflammatory macrophages (M1), which produce large amount of inflammatory cytokines like TNF- $\alpha$, IL-1 $\beta$, IL-6, IL-12, IL-18, and IL-23; and alternatively activated macrophages (M2) that produce other cytokines as IL-10, IL-1Ra, and IL-18BP ${ }^{[12]}$. M1 participate in polarized Th1 responses as inducers and effectors, while M2 support Th2-associated effects and functions ${ }^{[13]}$. Macrophages exhibit phenotypic plasticity to control immunological balance in the microenvironment ${ }^{[14]}$. However, the immunomodulatory effect of andrographolide on the alteration of macrophage phenotype has not been investigated. We studied how andrographolide altered macrophage phenotype in naïve and LPS/IL-4 activated macrophages by comparing the expression of activation-relevant M1 vs M2 cytokines.

Macrophages not only serve as the primary defense barrier in the innate immune response, but also act as important accessory cells in the adaptive immune response ${ }^{[15]}$. Activated macrophages phagocytose antigens by pattern recognition receptors (such as mannose receptor) ${ }^{[16]}$, and provided signals to mediate $\mathrm{T}$ cells activation. One signal is the major histocompatibility antigen complex binding to $\mathrm{T}$ cell receptor (TCR), the other is the interaction of co-stimulatory molecules with their respective ligands on $\mathrm{T}$ cells ${ }^{[17]}$. We observed effects of andrographolide on the antigen uptake and presenting capacity of macrophages by measuring mannose receptor (CD206), MHC I/II and co-stimulatory molecules (CD40, CD80, CD86) on naïve and LPS/IL-4 activated macrophages. In addition, yeast-derived recombinant HBsAg (vaccine qualified) was used as an antigen to induce Ag-specific antibody in a Th2/ M2-dependent mouse model of adaptive responses ${ }^{[18,19]}$, and effects of andrographolide on humor immune responses were evaluated by the levels of serum antibodies and the number of IL-4 producing splenocytes.

Studies on the signal transduction mechanism of macrophage activation have indicated that stimuli, such as LPS or IL-4, trigger the mitogenactivated protein kinase (MAPK) and phosphoinositol-3-kinase (PI3K) signaling pathways ${ }^{[20,21]}$. MAPK pathway positively regulated the expression of proinflammatory cytokines (TNF- $\alpha$, IL-1 $\beta$, IL-6 etc), whereas PI3K pathway conversely inhibits MAPK pathway activation and induced anti-inflammatory cytokines (IL-10 etc) ${ }^{[22,23]}$. To investigate the mechanisms of andrographolide regulating macrophage activation and polarization, the effects of andrographolide on phosphorylation level of ERK 1/2 (MAPK pathway) and AKT (PI3K pathway) were analyzed.

\section{Materials and methods Reagents}

Andrographolide was kindly provided by Chong Yuan Biology Science Co, Ltd (Nanjing, China), and it was dissolved in dimethyl sulfoxide (Sigma-Aldrich, St Louis, MO, USA) at 10 $\mathrm{mg} / \mathrm{mL}$ as a stock solution. LPS from $E$ coli strain 055:B5 was purchased from Sigma-Aldrich. Recombinant murine IL-4 and IL-13 were purchased from Pepro Tech Inc (Rocky Hill, NJ, USA). Human hepatitis B surface antigen (yeast-derived recombinant $\mathrm{HBsAg}$, vaccine qualified) was kindly provided by Beijing Institute of Biological Products (Beijing, China). Fluorochrome-labeled monoclonal antibodies to surface antigens on murine macrophages were purchased from eBioscience (San Diego, CA, USA). Antibodies against phospho-ERK, ERK, phospho-AKT, AKT and $\beta$-actin were purchased from Cell Signal Technology (Beverly, MA, USA).

\section{Endotoxin detection}

Contamination of endotoxin in andrographolide was assessed by the Limulus Amoebocyte Lysate (LAL) colorimetric assay (QCL- $1000^{\circledast}$ Endpoint LAL Assay; Lonza, Walkersville, MD, USA), according to the manufacturer's instructions. The absorbance at $405 \mathrm{~nm}$ is linear in the concentration range of 0.1-1.0 EU/mL endotoxin. The endotoxin concentration in diluted andrographolide was calculated with the absorbance from the standard curve by linear regression.

\section{Peritoneal macrophage isolation}

Primary mouse peritoneal macrophages were obtained from C57BL/ 6 mice (SPF, aged 6-8 weeks, SIPPR-BK Experimental Animal Ltd Co, Shanghai, China) 4 days after intraperitoneal injection of $1.5 \mathrm{~mL} 3 \%$ Brewer thioglycollate medium (Kang Run Biology Science Co Ltd, Shanghai, China). Peritoneal exudate cells were harvested from the peritoneal cavity of mice by lavage, washed with ice-cold RPMI-1640 medium (Invitrogen, Carlsbad, CA, USA) containing 1\% fetal bovine serum (FBS; Invitrogen), resuspended in pre-warmed RPMI-1640 containing $10 \%$ FBS and supplemented with $100 \mathrm{mg} / \mathrm{L}$ streptomycin and $10^{5} \mathrm{U} / \mathrm{L}$ penicillin (Invitrogen), and distributed in wells of the BD Falcon ${ }^{\mathrm{TM}}$ culture plates (BD Biosciences, San Jose, CA, USA). Macrophages were allowed to adhere for $2-3 \mathrm{~h}$ at $37^{\circ} \mathrm{C}$ in humidified atmosphere containing $5 \% \mathrm{CO}_{2}$, and nonadherent cells were removed by washing twice with PBS. Cell number and viability was assessed using trypan blue exclusion under microscope. Thioglycollate-treated mice will yield about $10^{7}$ peritoneal macrophages per mouse. Pooled macrophages were cultured and treated with andrographolide (10 $\mu \mathrm{g} / \mathrm{mL})$ and/or LPS/IL-4 (100 ng/mL). The purity of isolated mouse peritoneal macrophages pooled from five mice was greater than $95 \%$, as determined by staining with fluorescein isothiocyanate (FITC)-conjugated anti-mouse F4/80 and flow cytometric analysis.

\section{Macrophage viability}

Macrophage viability was assessed by the mitochondriadependent reduction of MTT, 3-(4,5-dimethyl-thiazol-2-yl)-2,5- 
diphenyltetrazolium bromide (Amresco, Solon, Ohio, USA) to formazan. In 96-well plates, $2 \times 10^{4}$ cells/well were treated with andrographolide and/or LPS/IL-4 for $24 \mathrm{~h}$, then MTT solution was added to a final concentration of $0.5 \mathrm{~g} / \mathrm{L}$ and further incubated for $4 \mathrm{~h}$ at $37^{\circ} \mathrm{C}, 5 \% \mathrm{CO}_{2}$. The culture medium was dumped off and $200 \mu \mathrm{L}$ DMSO was added to resuspend formazan into the solvent. The absorbance was measured at the test wavelength of $570 \mathrm{~nm}(670 \mathrm{~nm}$ as the reference wavelength) by the DTX Series Multimode Detectors (Beckman, Fullerton, CA, USA), and the percentage of viable cells was calculated versus untreated cell control.

\section{Macrophage cytokine gene expression}

Cytokine gene transcription of mouse peritoneal macrophages was measured by reverse transcription and real-time PCR. In 6-well plates, $4 \times 10^{6}$ cells/well were treated with andrographolide and/or LPS/IL-4 for $4 \mathrm{~h}$ or $24 \mathrm{~h}$. Total RNA was extracted using TRIzol reagent (Invitrogen) according to the manufacture's instruction, and treated with RNase-free DNase-I (Takara, Otsu, Shiga, Japan) for removal of potentially contaminating DNA. First-strand cDNA was synthesized from $2 \mu \mathrm{g}$ total RNA with random hexamer primer (Takara) and M-MLV reverse transcriptase (RT) (Promega, Madison, WI, USA) for $30 \mathrm{~min}$ at $37^{\circ} \mathrm{C}$. PCR amplification was performed using ABI PRISM ${ }^{\circledR} 7500$ real time PCR system (Applied Biosystems, Foster City, CA, USA), and reactions were carried out with $S Y B R^{\circledR}$ Premix Ex Taq ${ }^{\mathrm{TM}}$ ( $2 \times$ mixture) (Takara) and specific primers for mouse cytokines according to the sequences in GenBank (Table 1). Primers were designed using Beacon Designer 6.0 (PREMIER Biosoft International, Palo Alto, CA, USA) and synthesized by Sangon Biology Science

Table 1. Primer sequences for detecting mouse cytokine mRNA expression.

\begin{tabular}{|c|c|c|}
\hline Cytokine & $\begin{array}{l}\text { Accession number } \\
\quad(\text { mRNA) }\end{array}$ & Primer sequence $\left(5^{\prime} \rightarrow 3^{\prime}\right)$ \\
\hline TNF- $\alpha$ & NM_013693 & $\begin{array}{l}\text { f: GTG GAA CTG GCA GAA GAG } \\
\text { r: CCA TAG AAC TGA TGA GAG G }\end{array}$ \\
\hline IL-1 $\beta$ & NM_008361 & $\begin{array}{l}\text { f: TGG GAA ACA ACA GTG GTC AG } \\
\text { r: CCA TCA GAG GCA AGG AGG A }\end{array}$ \\
\hline IL-1Ra & NM_031167 & $\begin{array}{l}\text { f: ACA GTA GAA GGA GAC AGA AG } \\
\text { r: GGT GGT AGA GCA GAA GAC }\end{array}$ \\
\hline IL-6 & NM_031168 & $\begin{array}{l}\text { f: TGC CTT CTT GGG ACT GAT G } \\
\text { r: ACT CTG GCT TTG TCT TTC TTG T }\end{array}$ \\
\hline IL-10 & NM_010548 & $\begin{array}{l}\text { f: GAA GAC CCT CAG GAT GCG } \\
\text { r: CCA AGG AGT TGT TTC CGT TA }\end{array}$ \\
\hline IL-12 & NM_008352 & $\begin{array}{l}\text { f: AGA TGA AGG AGA CAG AGG AG } \\
\text { r: GCA CGA GGA ATT GTA ATA GC }\end{array}$ \\
\hline IL-18 & NM_008360 & $\begin{array}{l}\text { f: GAC TCT TGC GTC AAC TTC } \\
\text { r: GAT CAA TAT CAG TCA TAT CCT C }\end{array}$ \\
\hline IL-18BP & NM_010531 & $\begin{array}{l}\text { f: ACT TCT CCT GTT TGT TTG TG } \\
\text { r: TCT GGA TAC TGG GCT GTG }\end{array}$ \\
\hline GAPDH & NM_008084 & $\begin{array}{l}\text { f: GGT GAA GGT CGG TGT GAA CG } \\
\text { r: CTC GCT CCT GGA AGA TGG TG }\end{array}$ \\
\hline
\end{tabular}

f: forward primer; r: reverse primer.
Co, Ltd (Shanghai, China). Amplification conditions were: (1) $95^{\circ} \mathrm{C} / 10 \mathrm{~s}, 1$ cycle, for hot-start; (2) $95^{\circ} \mathrm{C} / 5 \mathrm{~s}, 60^{\circ} \mathrm{C} / 34 \mathrm{~s}$, 40 cycles, for PCR and data collection; (3) dissociation stage: $0.1^{\circ} \mathrm{C} / \mathrm{s}$ from 60 to $95^{\circ} \mathrm{C}$, for melting curve analysis. Relative quantification of gene expression in treatment groups $v s$ control (freshly isolated cells before culture) was calculated using the comparative threshold cycle method $2^{\wedge}\left[\left(\mathrm{Ct}_{\mathrm{GAPDH}}{ }^{-}\right.\right.$ $\left.\left.\mathrm{Ct}_{\mathrm{Gene}}\right)_{\text {treatment }}-\left(\mathrm{Ct}_{\mathrm{GAPDH}}-\mathrm{Ct}_{\mathrm{Gene}}\right)_{\text {control }}\right]$ where GAPDH is the housekeeping gene ${ }^{[24]}$.

\section{Macrophage cytokine production}

Mouse peritoneal macrophages $\left(4 \times 10^{6}\right.$ cells/well in 6 -well plate) were treated with andrographolide and/or LPS/IL-4/ IL-13 for $48 \mathrm{~h}$. Culture supernatants were collected, IL-12p40 and IL-10 concentrations were determined using specific ELISA kits (eBioscience), according to the manufacturer's instructions.

\section{Macrophage surface antigen expression}

Flow cytometry was used to determine the surface antigen expression (F4/80, MHC-I, MHC-II, CD40, CD80, CD86, and CD206) in mouse peritoneal macrophages. Briefly, $4 \times 10^{6}$ cells/well in 6-well plates treated with andrographolide and/ or LPS/IL-4 for $24 \mathrm{~h}$ were stained with fluorochrome-labeled mAbs (30 min on ice), washed with FACScan buffer (PBS containing $2 \%$ FBS and $0.1 \%$ Sodium Azide), and fixed with $4 \%$ paraformaldehyde. Flow cytometry acquisition was performed on FACScan ${ }^{\circledR}$ flow cytometer and data were analyzed using CellQuest ${ }^{\mathrm{TM}}$ software (BD Biosciences). The percentage of cells positive for each surface protein was determined based upon isotype control staining, and the amount of surface antigen expression of all cells counted was calculated by the following equation:

Expression Index $(\mathrm{EI})=\%$ positive cells $\times$ Mean Fluorescence Intensity ${ }^{[25]}$.

\section{Mice immunization and andrographolide administration}

Inbred female BALB/c mice (SPF, aged 6-8 weeks) were purchased from SIPPR-BK Lab Animal Co, Ltd (Shanghai, China) and housed under pathogen-free conditions. All animal studies were performed in accordance to national and international laws and policies. Mice were grouped as indicated in the experiments described below, each group consisting of 6 mice. Two groups of mice were immunized intramuscularly twice over a 4-week period with HBsAg alone ( $1 \mu \mathrm{g} /$ mouse) or followed by intraperitoneal administration of andrographolide $\left(1 \mathrm{mg} \cdot \mathrm{kg}^{-1} \cdot \mathrm{d}^{-1}, 7 \mathrm{~d}\right)$. Another group of mice received HBsAg admixed with $\mathrm{Al}(\mathrm{OH})_{3}$ adjuvant $\left[1 \mu \mathrm{g} \mathrm{HBsAg}+50 \mu \mathrm{g} \mathrm{Al}(\mathrm{OH})_{3} /\right.$ mouse] as a positive control. Naïve mice were used as a negative control.

\section{Detection of anti-HBsAg antibodies}

Sera were collected from the retroorbital plexuses of individual mice at 2, 4, and 6 weeks after the first HBsAg immunization. Anti-HBsAg antibodies were detected by anti-HBs ELISA kit (Diagnostic Reagent Center of Shanghai Municipal 
Infectious Diseases Hospital, Shanghai, China) according to the manufacturer's instruction. Each serum was diluted by serial threefold dilution and incubated in a 96-well plate precoated with HBsAg for $1 \mathrm{~h}$ at $37^{\circ} \mathrm{C}$. Plates were washed and horseradish peroxidase (HRP)-conjugated goat anti-mouse IgG (Huamei Bioengineering Co, Ltd, Shanghai) was added and further incubated for $1 \mathrm{~h}$ at $37^{\circ} \mathrm{C}$. After washing, the substrate (tetramethyl benzidine) was added and incubated for 15 $\mathrm{min}$ at $37^{\circ} \mathrm{C}$. The absorbance was measured at $450 \mathrm{~nm}$ with the reference wavelength of $630 \mathrm{~nm}$ in a 96-well plate spectrophotometer. Antibody titers are presented as the reciprocal of the highest dilution showing a positive reaction.

\section{Detection of IL-4-producing splenocytes}

After mice were sacrificed at 6 week after the first HBsAg immunization, spleens were removed and dissociated on 200-gauge nylon mesh. Splenocytes were treated with lysis buffer $\left(0.15 \mathrm{~mol} / \mathrm{L} \mathrm{NH}_{4} \mathrm{Cl}, 0.01 \mathrm{~mol} / \mathrm{L} \mathrm{KHCO}_{3}, 0.1 \mathrm{~mol} / \mathrm{L}\right.$ $\mathrm{Na}_{2}$ EDTA, $\mathrm{pH} 7.4$ ) at room temperature for $1.5 \mathrm{~min}$ to lyse red cells, washed with RPMI-1640 and resuspended in RPMI-1640 with 10\% FBS. IL-4-producing cells in spleens from immunized mice were detected by the enzyme-linked immunospot (ELISPOT) assay. Briefly, 96-well multiscreen filtration plates (Millipore, Billerica, MA, USA) were coated with anti-mouse IL- 4 capture antibody $(5 \mu \mathrm{g} / \mathrm{mL}$; BD Biosciences) overnight and blocked with culture medium for $1.5 \mathrm{~h}$. Spleen cells $\left(5 \times 10^{5}\right)$ were added to wells and stimulated with $0.5 \mu \mathrm{g} / \mathrm{mL} \mathrm{HBsAg}$ at $37^{\circ} \mathrm{C}$ for $48 \mathrm{~h}$. Plates were washed, and biotinylated anti-mouse IL-4 detection antibodies $(2 \mu \mathrm{g} / \mathrm{mL})$ were added and incubated for $2 \mathrm{~h}$ at room temperature. After washing, streptavidin-HRP was added and incubated for $1 \mathrm{~h}$ at room temperature, and 3-amino-9-ethylcarbazole was used as substrate. Spots were developed for about $30 \mathrm{~min}$ and the reaction was stopped by washing with deionized water. Spots were enumerated manually by inspection under a dissecting microscope, and automatically using an ImmunoSpot Analyzer-BioReader 4000 Pro-X (BIO-SYS GmbH, Karben, Germany).

\section{Detection of ERK 1/2 and AKT phosphorylation}

The phosphorylation level of ERK 1/2 and AKT proteins in mouse peritoneal macrophages was measured by Western blot assay. Briefly, in 6-well plates, $4 \times 10^{6}$ cells/well were treated with andrographolide and/or LPS/IL-4/IL-13 for $2 \mathrm{~h}$ or $12 \mathrm{~h}$. After washing with PBS, cells were lyzed in the lysis buffer (20 mmol/L Tris, pH 7.5, $150 \mathrm{mmol} / \mathrm{L} \mathrm{NaCl}, 1 \mathrm{mmol} / \mathrm{L} \mathrm{Na}_{2} \mathrm{E}-$ DTA, $2.5 \mathrm{mmol} / \mathrm{L}$ sodium pyrophosphate, $1 \%$ Triton $\mathrm{X}-100$, $1 \mathrm{mmol} / \mathrm{L} \beta$-glycerophosphate, $1 \mathrm{mmol} / \mathrm{L} \mathrm{Na}_{3} \mathrm{VO}_{4}, 1 \mu \mathrm{g} / \mathrm{mL}$ Leupeptin, $1 \mathrm{mmol} / \mathrm{L}$ PMSF) on ice and centrifuged $(14000 \times \mathrm{g}$, $5 \mathrm{~min}, 4^{\circ} \mathrm{C}$ ). The lysate of cells (mixed with $5 \times$ protein loading buffer and boiled for $5 \mathrm{~min}$ at $100{ }^{\circ} \mathrm{C}$ ) were analyzed by $12 \%$ SDS-PAGE, transferred to PVDF membrane (Millipore, Bedford, MA, USA) and blocked with 5\% nonfat milk in TBST buffer $(20 \mathrm{mmol} / \mathrm{L}$ Tris, $\mathrm{pH} 8.0,150 \mathrm{mmol} / \mathrm{L} \mathrm{NaCl}$ and $0.1 \%$ Tween-20) for $2 \mathrm{~h}$ at room temperature. The membranes were incubated with the primary antibody overnight at $4{ }^{\circ} \mathrm{C}$, then with a horseradish peroxidase-conjugated secondary antibody $1 \mathrm{~h}$ at room temperature, and detected by enhanced chemiluminescence kit (Thermo scientific, Waltham, MA, USA) according to the manufacturer's instructions. Protein molecular weight was estimated by Prestained Protein Ladder (Fermentas, Glen Burnie, MD, USA). For repeated immunoblotting, membranes were incubated in the stripping buffer (62.5 mmol/L Tris, pH 6.7, 20\% SDS and $100 \mathrm{mmol} / \mathrm{L} 2$-mercaptoethanol) for $30 \mathrm{~min}$ at $50{ }^{\circ} \mathrm{C}$.

\section{Statistical analysis}

Experimental data obtained were analyzed with the SPSS software. Variance between groups was analyzed by ANOVA, means of groups were compared by $t$-test. Differences with $P<0.05$ were considered statistically significant.

\section{Results}

\section{Endotoxin contamination of the andrographolide preparation}

The presence of endotoxin contamination in the preparations of test molecules is a major source of false results, in particular in assays on macrophages which are exquisitely sensitive to endotoxin stimulation. Thus, the presence of endotoxin in the andrographolide preparation was checked, by using a quantitative chromogenic LAL assay. Three concentrations of andrographolide were assayed $(10,50$, and $100 \mu \mathrm{g} / \mathrm{mL})$. At each of these concentrations the endotoxin contamination was below the detection limit of the assay $(0.1 \mathrm{EU} / \mathrm{mL})$. To make sure that andrographolide did not interfere with the enzymatic reaction at the basis of endotoxin detection, or with the optical reading of the yellow color developed by liberation of paranitroaniline, the reaction developed by known concentration of standard LPS $(0.5 \mathrm{EU} / \mathrm{mL})$ was compared with that developed by LPS in the presence of andrographolide. Addition of andrographolide does not affect in any way the detection of standard endotoxin, as shown in Figure 1.

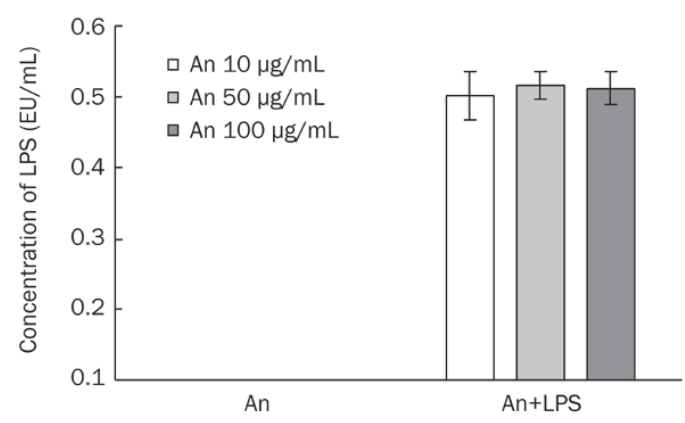

Figure 1. No endotoxin contamination in preparation of andrographolide (An). The An batch used for the experimental procedures was assessed at different concentrations $(10-100 \mu \mathrm{g} / \mathrm{mL})$ for endotoxin contamination (left part of the figure). As control of possible interference of An with the endotoxin-induced reaction, an dilutions were added to a fixed concentration of LPS $(0.5 \mathrm{EU} / \mathrm{mL})$ and tested in the LAL assay (right part of the figure). The lower detection limit of the assay is $0.1 \mathrm{EU} / \mathrm{mL}$. Endotoxin was not detected in An alone, and An did not inhibit detection of $0.5 \mathrm{EU} / \mathrm{mL}$ of standard LPS. 
Effects of andrographolide on murine macrophages viability

The in vitro toxicity of andrographolide and/or LPS or IL-4 on mouse peritoneal macrophages was assessed by MTT assay. At the concentration of $2.5-10 \mu \mathrm{g} / \mathrm{mL}$, andrographolide did not affect the viability of macrophages, but at the concentration of $40 \mu \mathrm{g} / \mathrm{mL}$, cell viability was inhibited by $50 \%$ versus the control (Figure 2). Therefore, the concentration of 10 $\mu \mathrm{g} / \mathrm{mL}$ was used for in vitro studies on macrophages.

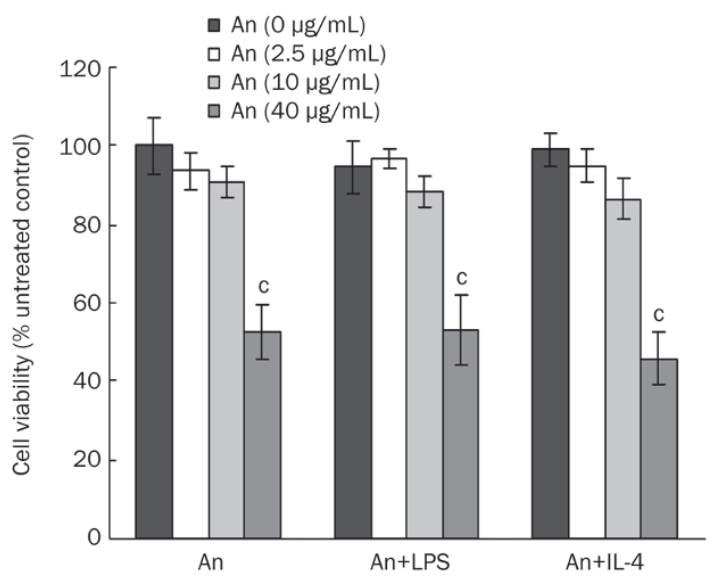

Figure 2. Effects of andrographolide (An) on macrophage viability. Mouse peritoneal macrophages $\left(2 \times 10^{4}\right.$ cells/well, 96-well plate) were incubated for $24 \mathrm{~h}$ with different concentrations of An $(0-40 \mu \mathrm{g} / \mathrm{mL})$ alone or together with LPS or IL-4 (both at $100 \mathrm{ng} / \mathrm{mL}$ ). Cell viability was determined using the MTT method, as described. Values are the mean $\pm S D$ of eight replicate wells from one experiment, representative of three performed. ${ }^{c} P<0.01$ vs no treatment control.

\section{Effects of andrographolide on cytokine expression in naive} murine macrophages

The effect of andrographolide on naive murine macrophage activation was evaluated by measuring expression of M1/M2 cytokine with quantitative real-time RT-PCR and ELISA assay (for IL-12 and IL-10). TNF- $\alpha$, IL-12, IL-1 $\beta$, IL-18, and IL-6 mRNA were measured as M1-related inflammatory cytokines, while IL-10, IL-1Ra, and IL-18BP were assessed as M2 activation cytokines. Treated with andrographolide for $24 \mathrm{~h}$, the mRNA level of both M1 and M2 cytokines in macrophages was decreased as compared to untreated cells: TNF-a, $25 \%$ of control; IL-12, 5\%; IL-13, 33\%; IL-10, 35\%; IL-1Ra, 25\% (Table $2)$. The results of M1/ M2 cytokine genes transcription were confirmed by IL-12 and IL-10 cytokine productions detected by ELISA, as shown in Figure 3.

Effects of andrographolide on cytokine expression in LPS/IL-4 activated murine macrophages

Murine macrophages were activated by LPS or IL-4 for M1 or M2 polarization. In LPS-activated macrophages, transcription of the cytokine genes detected as above was increased after $4 \mathrm{~h}$ of stimulation, while induced cytokine expression was partially restored upon longer stimulation time $(24 \mathrm{~h})$. In contrast,
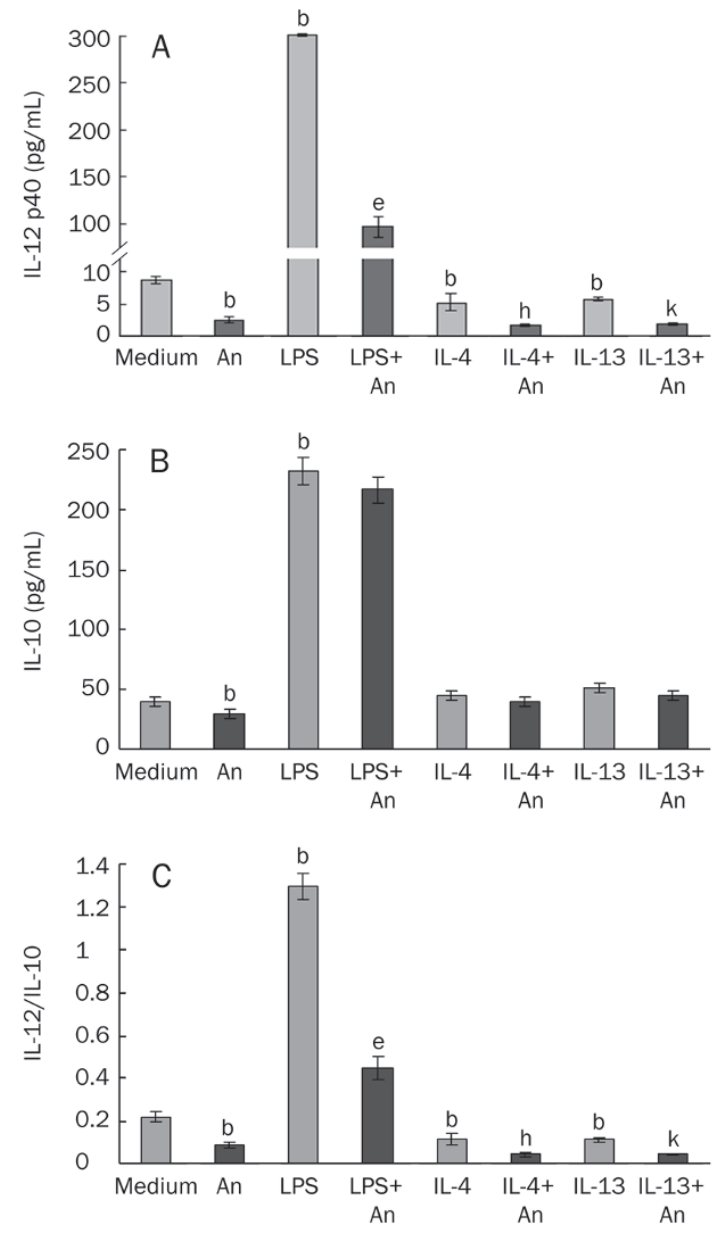

Figure 3. Effects of andrographolide (An) on IL-12 and IL-10 productions. Mouse peritoneal macrophages were incubated with An $(10 \mu \mathrm{g} / \mathrm{mL})$ alone or together with LPS, IL-4, or IL-13 (100 ng/mL) for $48 \mathrm{~h}$. IL-12 (A) and IL-10 (B) concentration in the supernatant were detected by ELISA, and the ratio of IL-12/IL-10 was calculated $(C)$. Values are the mean $\pm S D$ of three replicate wells from one experiment, representative of three performed. ${ }^{\mathrm{b}} P<0.05$ vs no treatment control; ${ }^{\mathrm{e}} P<0.05$ vs LPS treatment control; ${ }^{\mathrm{h}} P<0.05$ vs IL-4 treatment control; ${ }^{\mathrm{k}} P<0.05$ vs IL-13 treatment control.

the expression of the M1 cytokine (TNF-a) was decreased and that of the M2 cytokine (IL-10) was increased in IL-4-activated macrophages (Table 2).

The effect of andrographolide on LPS/IL-4 activated murine macrophages was evaluated by measuring expression of activation relevant cytokines. When andrographolide was added along with LPS or IL-4, it down-regulated expression of both M1 and M2 cytokines in activated macrophages, shown in Table 2 .

It is important to note that the most important parameters for discriminating between M1 and M2 polarization are the relative levels of IL-12 vs IL-10 expression. M1 macrophages typically express high level of IL-12 and low level of IL-10, and M2 cells vice versa. Thus, the IL-12/IL-10 ratio has been calculated for untreated macrophages $(0.488$ at 4 and 0.789 at $24 \mathrm{~h}$ ), for cells treated with LPS (31.0 at $4 \mathrm{~h}$ and 9.02 at $24 \mathrm{~h}$, 
Table 2. The effects of andrographolide (An) on cytokine mRNA expression level in naive and activated macrophages.

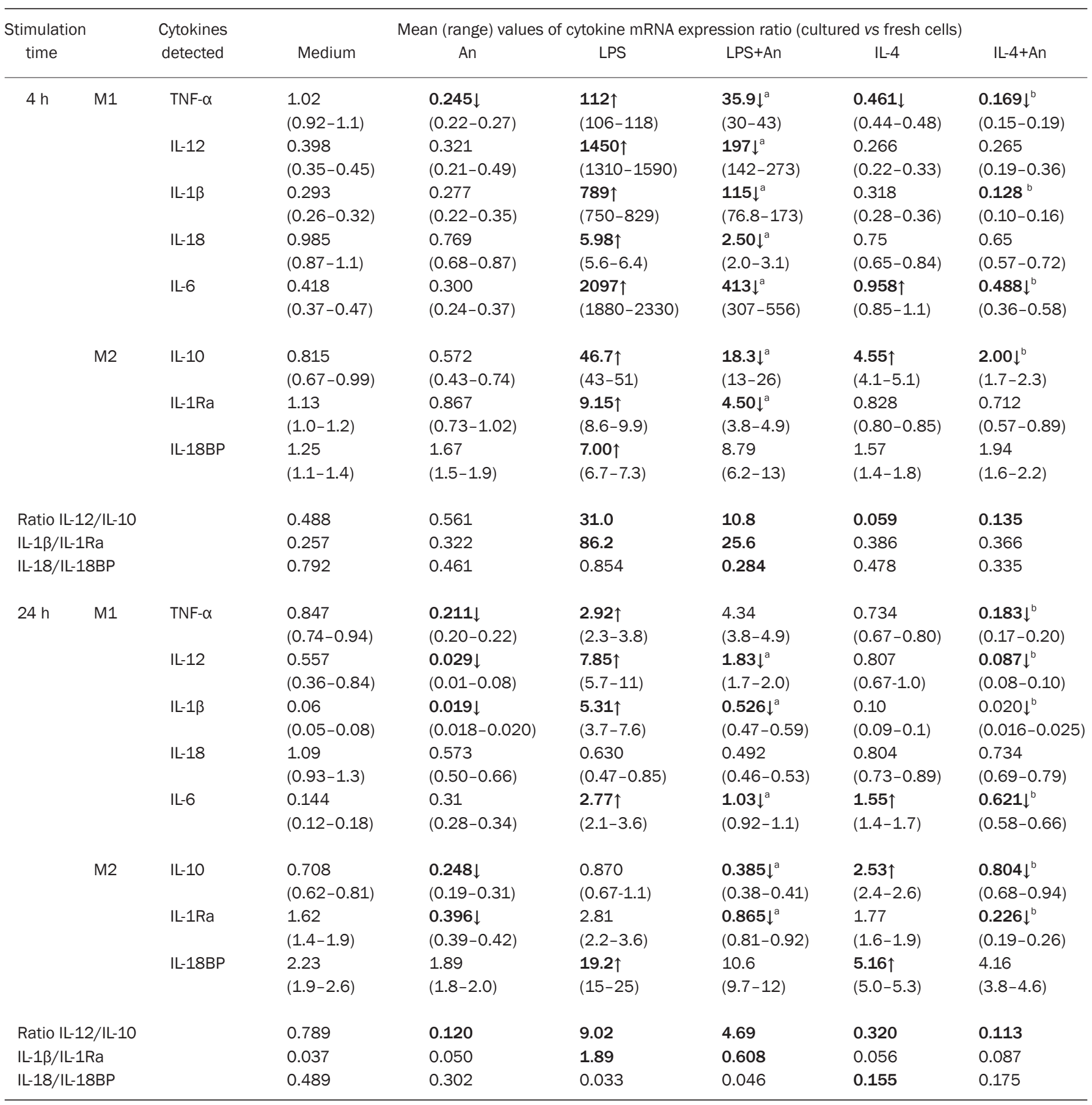

Data are shown in the table as $2^{-\Delta \Delta \mathrm{Ctmean}}\left[2^{-(\Delta \Delta \mathrm{Ct}+\mathrm{SD})}, 2^{-(\Delta \Delta \mathrm{Ct}-\mathrm{SD})}\right], \Delta \Delta \mathrm{Ct}=\left(\mathrm{Ct}_{\mathrm{Gene}}-\mathrm{Ct}_{\mathrm{GAPDH}}\right)_{\text {treatment }}-\left(\mathrm{Ct}_{\mathrm{Gene}}-\mathrm{Ct}_{\mathrm{GAPDH}}\right)_{\text {control }}$.

Data in bold are those with $>2$-fold difference. $\uparrow$ up-regulation ( $>2$-fold increase vs medium control), $\downarrow$ down-regulation ( $>2$-fold decrease vs medium control),

$\downarrow^{\mathrm{a}}$ down-regulation (>2-fold decrease vs LPS-treated control), $\downarrow^{\mathrm{b}}$ down-regulation (>2-fold decrease vs IL-4-treated control).

indicating strong M1 polarization), and for IL-4-treated cells ( 0.059 and 0.320 at 4 and $24 \mathrm{~h}$ ie distinctly M2). Treatment with andrographolide could polarize M2 direction in the control cells after $24 \mathrm{~h}$ of exposure (IL-12/IL-10 ratio from 0.789 to 0.120), strongly decrease M1 polarization of LPS-treated cells (from 31.0 to 10.8) at $4 \mathrm{~h}$, and further increase M2 phenotype in IL-4 treated cells (from 0.320 to 0.113 ) (Table 2). The results of M1/M2 cytokine genes transcription were confirmed by IL-12 and IL-10 cytokine productions detected by ELISA, as shown in Figure 3. 
Effects of andrographolide on surface antigen expression in murine macrophages

To evaluate the effect of andrographolide on the antigen uptake and presenting capacity of macrophages, modulation of mannose receptor (CD206), MHC class I/II and co-stimulating molecules (CD40, CD80, CD86) was assessed by flow cytometry in naïve macrophages or cells activated with LPS or IL-4 in the presence or in the absence of andrographolide.

When murine macrophages were treated by andrographolide alone, the expression of MHC-I (EI 24.9) was downregulated comparing with basal antigen expression in naïve macrophages (MHC-I: 47.5; MHC-II: 0.157; CD40: 9.86; CD80: 9.90; CD86: 3.57; CD206: 4.25). Activation with LPS increased the expression of MHC-I (189), CD40 (60.3), CD80 (18.3) and CD86 (10.8). However, activation with IL-4 increased the expression of CD40 (17.4) and CD206 (19.7), as shown in Figure 4.

When andrographolide was added along with LPS, it downregulated the LPS-induced increase of MHC-I (from 189 to 103), CD40 (from 60.3 to 27.1), CD80 (from 18.3 to 6.94) and CD86 (from 10.8 to 4.23 ). Andrographolide treatment along with IL-4 down-regulated IL-4-induced expression of CD40
$\mathrm{MHC}$ I
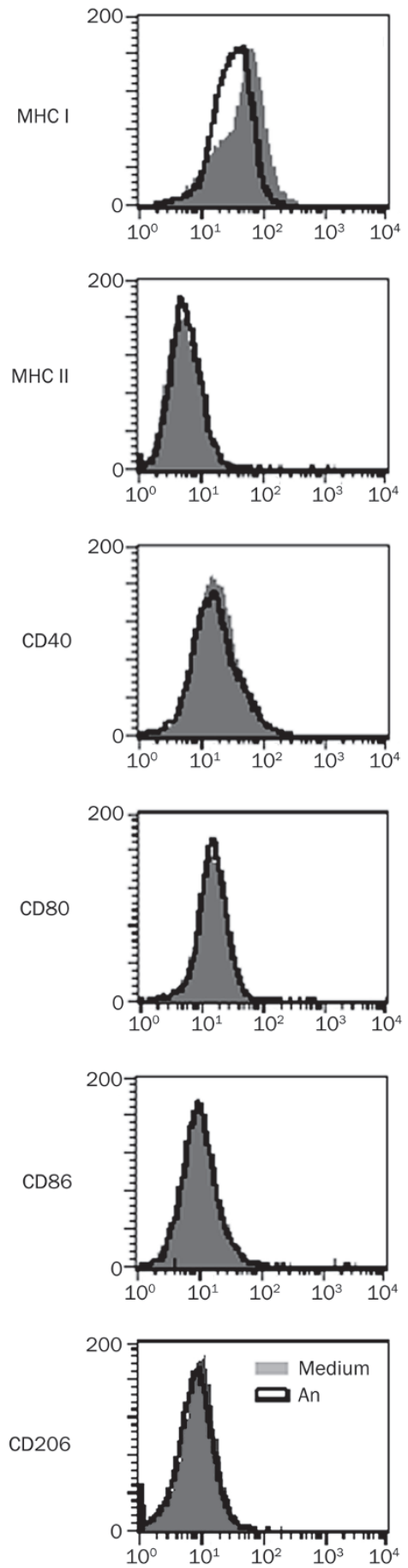
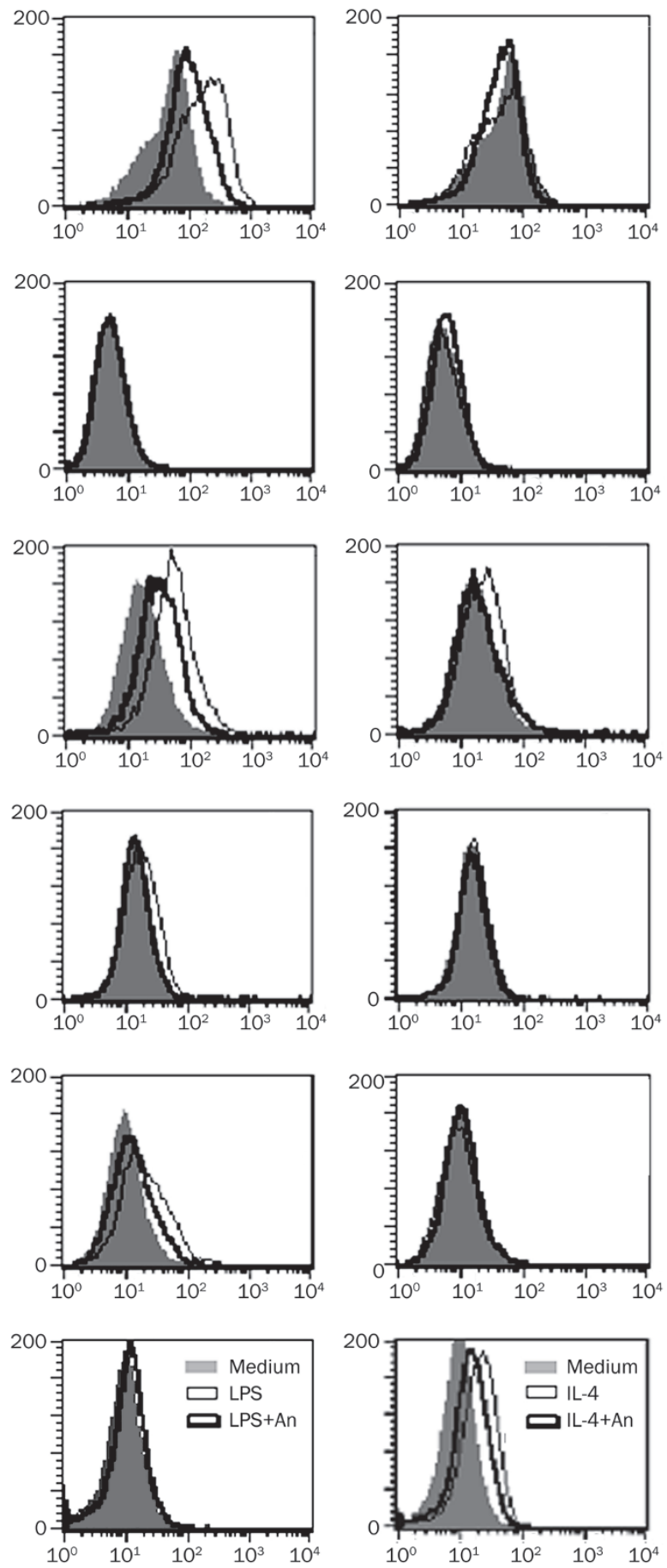

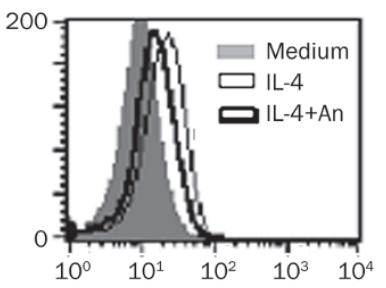

Figure 4. Effect of andrographolide (An) on macrophage surface antigen expression. Macrophages $\left(4 \times 10^{6}\right.$ cells/ well, 6-well plate) were incubated for $24 \mathrm{~h}$ with An $(10 \mu \mathrm{g} / \mathrm{mL})$ alone or together with LPS or IL-4 (both at $100 \mathrm{ng} / \mathrm{mL}$ ). Expression of surface antigens was tested by flow cytometric analysis, as described. Data were analysed by CellQuest ${ }^{\mathrm{TM}}$ software. Data from one experiment representative of three performed. 
(from 17.4 to 14.2) and CD206 (from 19.7 to 11.6), as shown in Figure 4.

\section{Effects of andrographolide on specific antibody response}

To determine whether andrographolide can regulate the adaptive immune response in vivo, its effect on specific antibody response was tested in mice vaccinated with HBsAg. Mice were primed intramuscularly with $\mathrm{HBsAg}(1 \mu \mathrm{g} /$ mouse $)$, and andrographolide was administered intraperitoneally for 7 consecutive days starting from the day of priming. After 4 weeks the mice were boosted with the same dose of HBsAg. Mice immunized with HBsAg plus alum was used as control. AntiHBsAg antibody titers were determined at 2 and 4 weeks after priming, and 2 weeks after boosting (ie, 6 weeks after priming). Treatment of andrographolide resulted in decreased anti-HBs titers at all time tested (Figure 5A). At 2 weeks after boosting, splenocytes were isolated from immunized mice, stimulated in vitro with HBsAg $(0.5 \mu \mathrm{g} / \mathrm{mL})$, and IL-4-producing cells were measured by ELISPOT. The number of IL-4-producing cells in the spleen of HBsAg-immunized mice $\left(23 / 10^{6}\right.$ spleen cells)
A

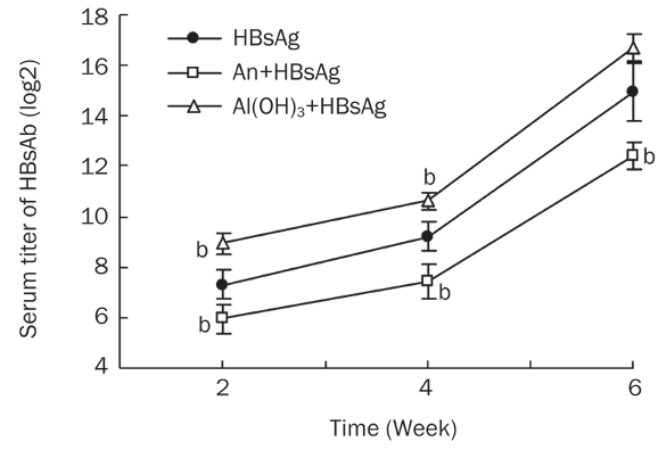

B

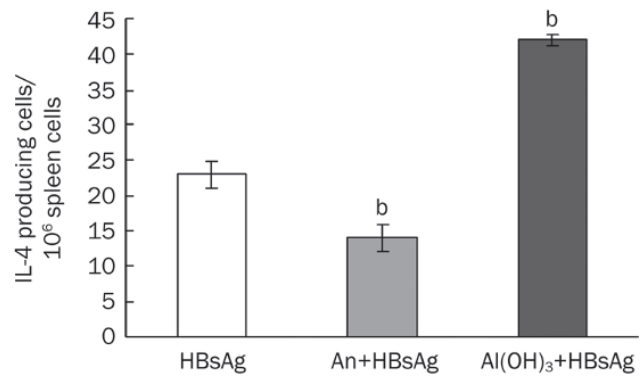

Figure 5. Andrographolide (An) inhibits serum anti-HBsAg antibody titers and reduces the numer of IL-4-producing cells. Mice were immunized with $\mathrm{HBsAg}$ as described. The first group received $\mathrm{HBsAg}$ alone (two administrations of $1 \mu \mathrm{g} /$ mouse 4 weeks apart). The second group received $\mathrm{HBsAg}$ and repeated administrations of $\mathrm{An}\left(1 \mathrm{mg} \cdot \mathrm{kg}^{-1} \cdot \mathrm{d}^{-1}, 7 \mathrm{~d}\right)$. The last group received $\mathrm{HBsAg}$ plus $\mathrm{Al}(\mathrm{OH})_{3}$ adjuvant (50 $\mu \mathrm{g} /$ mouse). (A) Serum anti-HBsAg antibodies were measured by ELISA after 2, 4, and 6 weeks from the first antigen inoculum. Antibody titers are presented as the reciprocal of the highest dilution showing a positive reaction. (B) At 6 weeks ( 2 weeks after the second antigen inoculum) mice were sacrificed, spleen cells isolated and challenged in culture with HBsAg $(0.5 \mu \mathrm{g} /$ $\mathrm{mL}$ ). The number of IL-4-producing cells $/ 10^{6}$ spleen cells was measured by mouse IL-4 ELISPOT. Data are presented as the mean \pm SD of values from 6 mice/group in one experiment, representative of three performed. ${ }^{\mathrm{b}} \mathrm{P}<0.05$ vs the control group of HBsAg. was decreased by $40 \%$ in mice immunized with HBsAg plus andrographolide, while in mice immunized with $\mathrm{HBsAg}$ plus alum IL-4-producing cells increased about 2 fold (Figure 5B).

Effects of andrographolide on ERK 1/2 and AKT phosphorylation As MAPK and PI3K signaling pathway are involved in the macrophage activation, the effects of andrographolide on phosphorylation level of ERK 1/2 (MAPK pathway) and AKT (PI3K pathway) were analyzed by Western blot assay in naïve macrophages or cells activated with LPS or IL-4/IL-13 in the presence or in the absence of andrographolide. Activation by LPS or IL-4/IL-13 increased ERK 1/2 and AKT phosphorylation. Treatment with andrographolide for $2 \mathrm{~h}$ exerted an apparent inhibitory effect on phosphorylation of ERK $1 / 2$ and AKT in both M1 and M2 activated macrophages. However, this modulatory effect of andrographolide was reversed or restored after $12 \mathrm{~h}$ treatment (Figure 6, 7).

\section{Discussion}

Macrophages have remarkable heterogeneity and plasticity that allows them to efficiently respond to exogenous and endogenous signals by changing their functional phenotype ${ }^{[11,14]}$. In this study, thioglycollate-elicited mouse peritoneal macrophage $\left(\mathrm{F} 4 / 80^{+} \text {population }\right)^{[26]}$ have been used to investigate the immunomodulatory activities of andrographolide on the innate immune response in vitro. Our results indicated that andrographolide showed potent immunoregulatory properties in murine macrophages. Andrographolide reduced expression of inflammatory cytokines (TNF-a, IL-12, IL-1 $\beta$, IL-18, and IL-6) in LPS activated macrophages, which is consistent with a previous report that andrographolide decreased TNF- $\alpha$ and IL-12 mRNA expression and cytokine production in murine peritoneal macrophages, although they did not detect IL-1 $\beta$, IL-18, and IL-6 ${ }^{[27]}$.

The response of macrophages to the microenvironment changes is of key importance in the ability of the organism to cope with dangers (eg an infection). For example, at the acute stage of an infection, macrophages respond to microbial exogenous signals such as LPS (a structural component of Gramnegative bacteria) and produce pro-inflammatory cytokines (classical inflammatory activation). This reaction is important for the efficient control of growth and dissemination of invading pathogens. At later times, other signals such as the endogenous anti-inflammatory cytokine IL-4/IL-13 can re-direct macrophage activation into an alternative regulatory pathway (alternative M2 activation) that promotes the resolution of inflammation by reducing inflammatory cytokine production and inducing synthesis of anti-inflammatory and tissue repairing factors ${ }^{[12,28]}$. We observed the phenomenon in this study that LPS induced a rapid and significant increase of cytokines gene transcription at the early time $(4 \mathrm{~h})$, which returns toward background at $24 \mathrm{~h}$. On the other hand IL-4 did not up-regulate inflammatory cytokine expression but was able to induce expression of regulatory molecules IL-10 and IL-18BP also at the late time, and this tendency was also observed in IL-13 treated macrophages (Supplementary Table 1). 

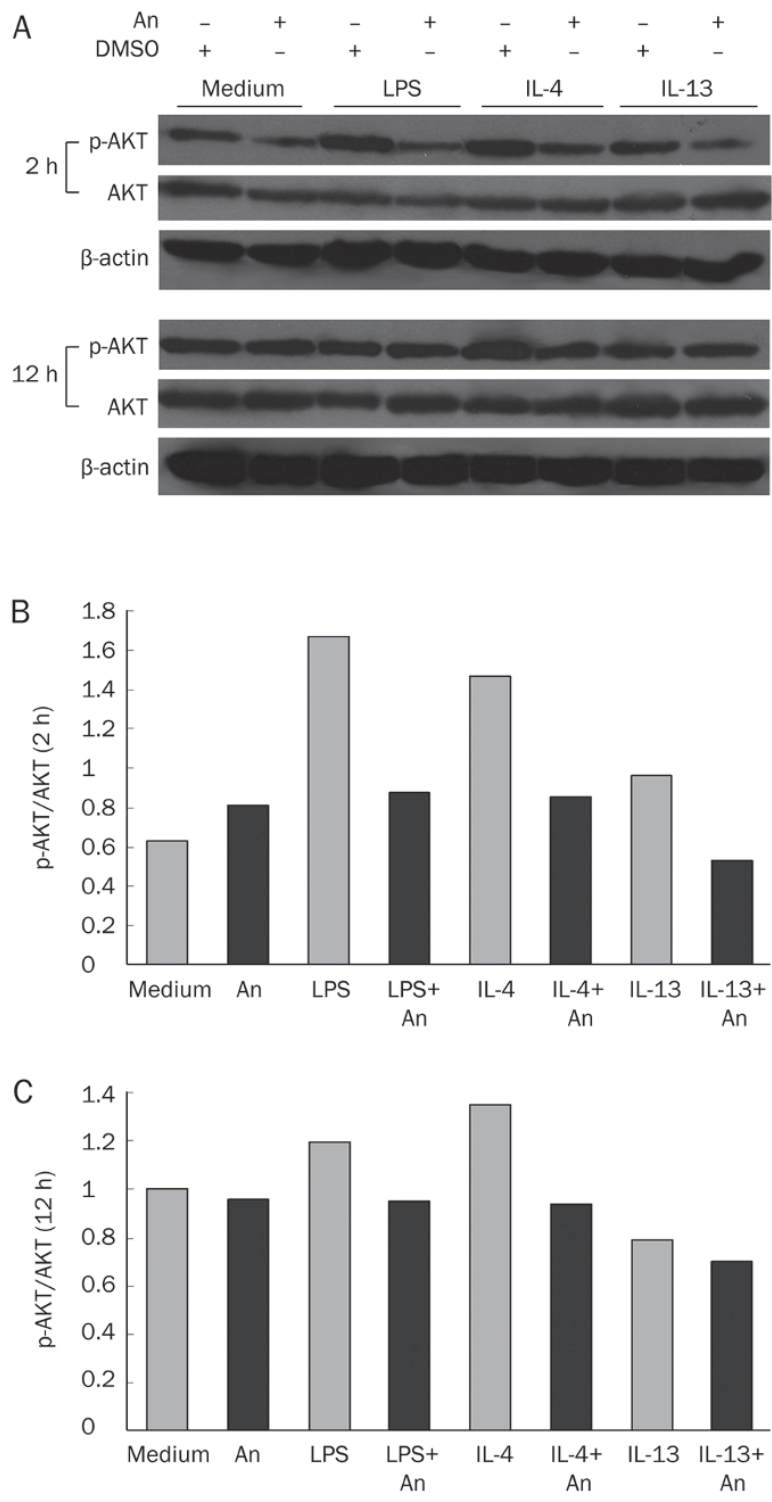

Figure 6. Andrographolide (An) modulates the PI3K signalling pathway activation. Macrophages were incubated with $A n(10 \mu \mathrm{g} / \mathrm{mL})$ alone or together with LPS, IL-4 or IL-13 for $2 \mathrm{~h} / 12 \mathrm{~h}$. (A) AKT phosphorylation and total ERK $1 / 2$ expression were detected by Western Blot. $\beta$-actin was used as a protein loading control. (B, C) The densitometric analysis of band intensity in (A) is analyzed by Quantity One software (Bio-Rad).

It has been described that a phenotypic switch in the macrophage population occurs over time in some cases and is associated with immune mediated pathology ${ }^{[11]}$. On one hand, over-production of inflammatory cytokines can lead to chronic inflammation and a variety of autoimmune diseases, including inflammatory bowel disease, rheumatoid arthritis, asthma and even atherosclerosis. On the other hand, over-production of anti-inflammatory cytokines can lead to persistent infection and tumor tolerance ${ }^{[29,30]}$. Regulation of macrophage activation by immunomodulatory drugs at specific stage of different diseases can be of important therapeutic benefit. In the present study, LPS or IL-4 activated mouse peritoneal
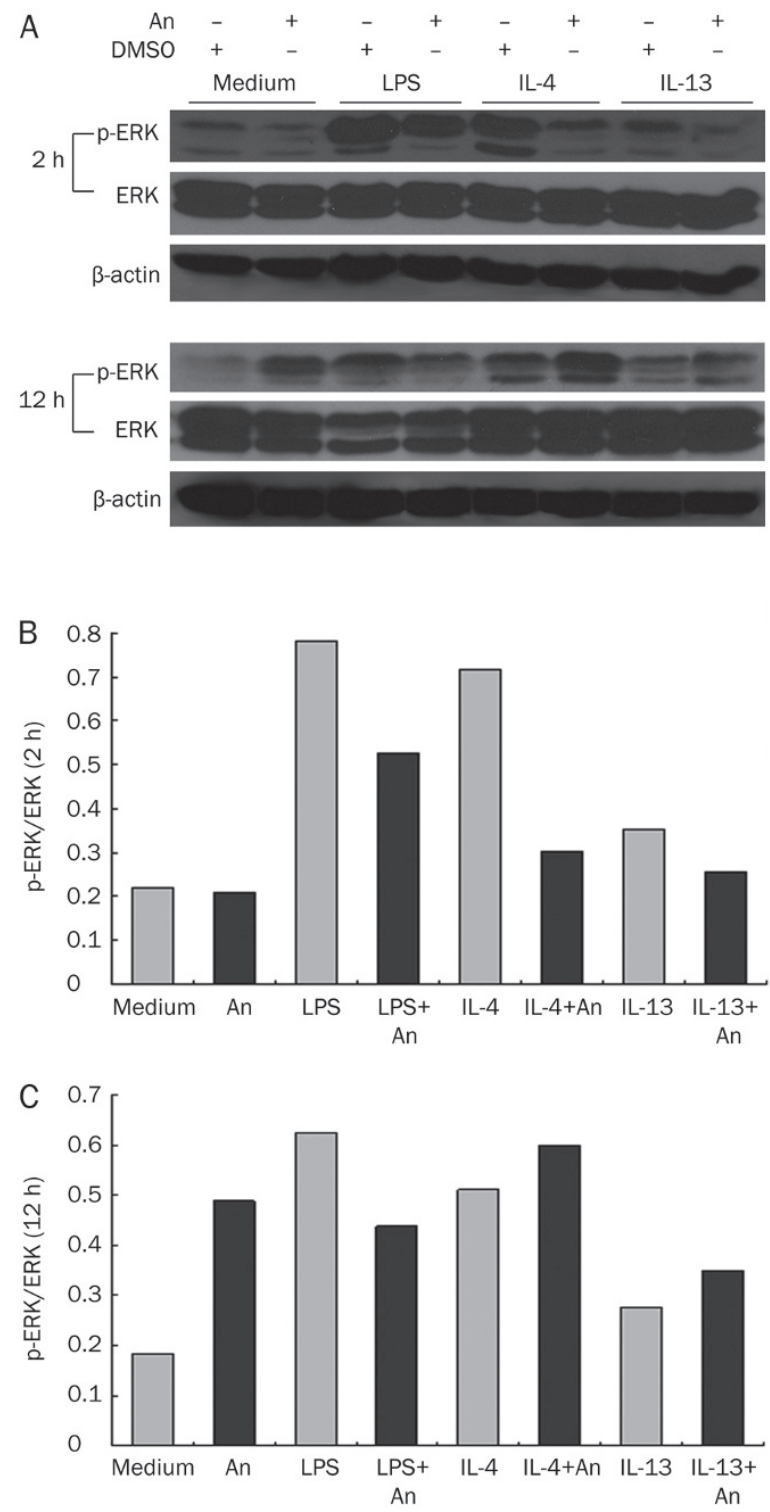

Figure 7. Andrographolide (An) modulates the MAPK signalling pathway activation. Macrophages were incubated with $A n(10 \mu \mathrm{g} / \mathrm{mL})$ alone or together with LPS, IL-4 or IL-13 for $2 \mathrm{~h} / 12 \mathrm{~h}$. (A) ERK $1 / 2$ phosphorylation and total AKT expression were detected by Western Blot. $\beta$-actin was used as a protein loading control. (B, C) The densitometric analysis of band intensity in (A) is analyzed by Quantity One software (Bio-Rad).

macrophages were treated with andrographolide, to assess the effect of the agent in regulating macrophage activation. It showed that andrographolide significantly down regulated the expression of both M1- and M2-related cytokines in macrophages activated with either LPS or IL-4. As both inflammatory and anti-inflammatory cytokine genes are triggered by LPS and IL-4 (as prototypes of type 1 and type 2 inflammation), the relative activation of inflammatory vs antiinflammatory genes is a better estimate of M1 vs M2 polarization as compared to assessement of single genes. The ratio of M1:M2 cells may be more important than the absolute number 
of cells $^{[31]}$. M1 macrophage cells usually have the phenotype showing IL-12 high, IL-10 low, IL-23 high, and M2 cells vice versa $^{[32]}$. The ratio between IL-12 and IL-10 production is indicative of classical $v$ s alternative activation. We found that treatment of andrographolide for $4 \mathrm{~h}$ significantly decreased the LPS-induced M1 polarization (IL-12/IL-10 ratio from 31.0 to 10.8) and slightly reversed the IL-4-induced M2 polarization (from 0.059 to 0.135 ). After $24 \mathrm{~h}$ of treatment andrographolide showed anti-inflammatory effect on LPS-stimulated cells (IL-12/IL-10 ratio from 9.02 to 4.69), but tended toward the M2-like phenotype of IL-4-activated cells (from 0.320 to 0.113). The inhibitory activity of andrographolide on IL-23 was also observed in LPS-induced macrophages (Supplementary Table 2). Correspondingly, IL-12 production and the ratio of IL-12/IL-10 were reduced by andrographolide after $48 \mathrm{~h}$ of treatment. Thus andrographolide acted as a modulator in macrophage activation depending on the initial microenvironment.

Cytokine environment and cell-cell interaction are important factors in the adaptive immune response. As professional antigen-presenting cells, macrophages not only respond to Th1 and Th2 cytokines and alter their functional phenotypes, but also secrete different amounts of M1 and M2 cytokines and express antigen uptake and presenting related surface markers (such as mannose receptor, MHC and costimulatory molecules) to present antigen protein and activate T/B cells ${ }^{[13,33]}$. The effects of andrographolide were thus investigated on the antigen-uptake and presenting capacity related suface markers of macrophages in vitro and specific antibody response in vivo. Andrographolide inhibited LPS-induced expression of class I MHC and costimulatory molecules (CD40, CD80, and CD86), and inhibited IL-4 induced expression of mannose receptor. Similar effects have been observed in dendritic cells, in which andrographolide inhibited LPS-induced up-regulation of the maturation markers CD40 and CD86 ${ }^{[9]}$. Administration of andrographolide to mice immunized with HBsAg (vaccine quality) significantly down-regulated anti-HBs production. The capacity of andrographolide to reduce macrophage expression of pattern recognition receptor (mannose receptor, CD206) and $\mathrm{MHC} /$ costimulatory molecules indicated that andrographolide may inhibit the antigen uptake and presentation and result in reduced specific antibody production. HBsAg induces Th2 biased response and increases specific IL4-producing splenocytes after immunization of mice ${ }^{[19]}$. Treatment with andrographolide decreased the number of HBsAg specific IL-4-producing cells in parallel to the decrease of antiHBs production.

MAPK and PI3K signaling pathway have been reported to be involved in macrophage activation ${ }^{[22,23]}$, the effects of andrographolide on the signaling pathway activation were analyzed by detecting phosphorylation level of ERK 1/2 (MAPK pathway) and AKT (PI3K pathway). Andrographolide exerted an apparent inhibitory effect on phosphorylation of ERK $1 / 2$ and AKT at the early time $(2 \mathrm{~h})$ and reversed or restored them after $12 \mathrm{~h}$, suggesting that ERK $1 / 2$ and AKT pathways may contribute to the regulatory activity of andrographolide on macrophage activation. The inhibitory effect of andrographolide on PI3K and MAPK pathway may indicate that the mechanism of andrographolide modulating macrophage polarization is not the direct activation of naïve macrophages but an inhibitory effect on already activated macrophages.

The results indicate that andrographolide is able to modulate the innate immune response by regulating both classical and alternative activation of macrophages, and regulate specific antibody production as well as antigen-specific IL-4 producing splenocytes. Our study provides more evidences for using andrographolide as an immunomodulatory drug in the therapy for immune disorders.

\section{Acknowledgements}

This work was supported by the Program of Ministry of Science and Technology of China (S2010GR0385, 2008ZX10004014, and 2008ZX10002-002), the International Science and Technology Cooperation Program of Shanghai Science and Technology Commission (074307038) and PhD student visiting scholarship of Fudan University for 2008-2009.

\section{Author contribution}

Prof Di QU and Prof Shi-ping MA designed the project and edited the manuscript. Prof Diana BORASCHI made suggestions for the project and manuscript. Wei WANG and Jing WANG designed and performed and analyzed most of the studies and wrote the manuscript. Sheng-fu DONG and Chun-hong LIU performed the research and analysis of experiments with mice immunization and antibody detection. Paola ITALIANI performed the research and analysis of experiments with endotoxin detection. Shu-hui SUN and Jing XU contributed new reagents and analytical performance of flow cytometry.

\section{Supplementary informaiton}

Supplementary Table 1 show the effects of andrographolide (An) on M1/ M2 cytokines mRNA expression level in IL-13 activated macrophages. Supplementary Table 2 show the effects of andrographolide (An) on IL-23 mRNA expression level in naive and activated macrophages. Supplementary data associated with this article can be found in the online version.

\section{References}

1 State Pharmacopoeia Committee. Chinese Pharmacopoeia. Beijing: Chemical Industry Press; 2005. p 189-190.

2 Kligler B, Ulbricht C, Basch E, Kirkwood CD, Abrams TR, Miranda M, et al. Andrographis paniculata for the treatment of upper respiratory infection: A systematic review by the natural standard research collaboration. Explore-Ny 2006; 2: 25-9.

3 Abu-Ghefreh AA, Canatan $\mathrm{H}$, Ezeamuzie $\mathrm{Cl}$. In vitro and in vivo antiinflammatory effects of andrographolide. Int Immunopharmacol 2009; 9: 313-8.

4 Zhou J, Lu GD, Ong CS, Ong CN, Shen HM. Andrographolide sensitizes cancer cells to TRAIL-induced apoptosis via p53-mediated death receptor 4 up-regulation. Mol Cancer Ther 2008; 7: 2170-80.

5 Yu BC, Chang CK, Su CF, Cheng JT. Mediation of beta-endorphin in 
andrographolide-induced plasma glucose-lowering action in type I diabetes-like animals. Naunyn-Schmiedeberg's Arch Pharmacol 2008; 377: 529-40.

6 Woo AY, Waye MM, Tsui SK, Yeung ST, Cheng CH. Andrographolide up-regulates cellular-reduced glutathione level and protects cardiomyocytes against hypoxia/reoxygenation injury. J Pharmacol Exp Ther 2008; 325: 226-35.

7 Sheeja K, Kuttan G. Modulation of natural killer cell activity, antibodydependent cellular cytotoxicity, and antibody-dependent complementmediated cytotoxicity by andrographolide in normal and Ehrlich ascites carcinoma-bearing mice. Integr Cancer Ther 2007; 6: 66-73.

8 Sheeja K, Kuttan G. Activation of cytotoxic T lymphocyte responses and attenuation of tumor growth in vivo by Andrographis paniculata extract and andrographolide. Immunopharmacol Immunotoxicol 2007; 29: 81-93.

9 Iruretagoyena MI, Tobar JA, Gonzalez PA, Sepulveda SE, Figueroa CA, Burgos RA, et al. Andrographolide interferes with T cell activation and reduces experimental autoimmune encephalomyelitis in the mouse. J Pharmacol Exp Ther 2005; 312: 366-72.

10 Iruretagoyena MI, Sepulveda SE, Lezana JP, Hermoso M, Bronfman M, Gutierrez MA, et al. Inhibition of nuclear factor-kappa B enhances the capacity of immature dendritic cells to induce antigen-specific tolerance in experimental autoimmune encephalomyelitis. J Pharmacol Exp Ther 2006; 318: 59-67.

11 Mosser DM, Edwards JP. Exploring the full spectrum of macrophage activation. Nat Rev Immunol 2008; 8: 958-69.

12 Martinez FO, Sica A, Mantovani A, Locati M. Macrophage activation and polarization. Front Biosci 2008; 13: 453-61.

13 Mills CD, Kincaid K, Alt JM, Heilman MJ, Hill AM. M-1/M-2 macrophages and the Th1/Th2 paradigm. J Immunol 2000; 164: 6166-73.

14 Zhang X, Mosser DM. Macrophage activation by endogenous danger signals. J Pathol 2008; 214: 161-78.

15 Bowdish DM, Loffredo MS, Mukhopadhyay S, Mantovani A, Gordon S. Macrophage receptors implicated in the "adaptive" form of innate immunity. Microbes Infect 2007; 9: 1680-7.

16 Gazi U, Martinez-Pomares L. Influence of the mannose receptor in host immune responses. Immunobiology 2009; 214: 554-61.

17 Crow MK. Modification of accessory molecule signaling. Springer Semin Immunopathol 2006; 27: 409-24.

18 Jafarzadeh A, Zarei S, Shokri F. Low dose revaccination induces robust protective anti-HBs antibody response in the majority of healthy non-responder neonates. Vaccine 2008; 26: 269-76.

19 Dong SF, Chen JM, Zhang W, Sun SH, Wang J, Gu JX, et al.
Specific immune response to HBsAg is enhanced by beta-glucan oligosaccharide containing an alpha-(1-->3)-linked bond and biased towards M2/Th2. Int Immunopharmacol 2007; 7: 725-33.

20 Gwinn MR, Vallyathan V. Respiratory burst: role in signal transduction in alveolar macrophages. J Toxicol Environ Health B Crit Rev 2006; 9: 27-39.

21 Varin A, Gordon S. Alternative activation of macrophages: Immune function and cellular biology. Immunobiology 2009; 214: 630-41.

22 Rao KM. MAP kinase activation in macrophages. J Leukoc Biol 2001; 69: 3-10.

23 Fukao T, Koyasu S. PI3K and negative regulation of TLR signaling. Trends Immunol 2003; 24: 358-63.

24 Livak KJ, Schmittgen TD. Analysis of relative gene expression data using real-time quantitative PCR and the 2(-Delta Delta $C(T)$ ) method. Methods 2001; 25: 402-8.

25 D'Anto V, Eckhardt A, Hiller KA, Spagnuolo G, Valletta R, Ambrosio L, et al. The influence of $\mathrm{Ni}(\mathrm{II})$ on surface antigen expression in murine macrophages. Biomaterials 2009; 30: 1492-501.

26 Gordon S. The macrophage: past, present and future. Eur J Immunol 2007; 37 Suppl 1: S9-17.

27 Qin LH, Kong L, Shi GJ, Wang ZT, Ge BX. Andrographolide inhibits the production of TNF-alpha and interleukin-12 in lipopolysaccharidestimulated macrophages: role of mitogen-activated protein kinases. Biol Pharm Bull 2006; 29: 220-4.

28 Bouhlel MA, Derudas B, Rigamonti E, Dievart R, Brozek J, Haulon S, et al. PPARgamma activation primes human monocytes into alternative M2 macrophages with anti-inflammatory properties. Cell Metab 2007; 6: 137-43.

29 Mosser DM. The many faces of macrophage activation. J Leukoc Biol 2003; 73: 209-12.

30 Mora AL, Torres-Gonzalez E, Rojas M, Corredor C, Ritzenthaler J, Xu J, et al. Activation of alveolar macrophages via the alternative pathway in herpesvirus-induced lung fibrosis. Am J Respir Cell Mol Biol 2006; 35: 466-73.

31 Brown BN, Valentin JE, Stewart-Akers AM, McCabe GP, Badylak SF. Macrophage phenotype and remodeling outcomes in response to biologic scaffolds with and without a cellular component. Biomaterials 2009; 30: 1482-91.

32 Mantovani A, Sica A, Locati M. Macrophage polarization comes of age. Immunity 2005; 23: 344-6.

33 Ma J, Chen T, Mandelin J, Ceponis A, Miller NE, Hukkanen M, et al. Regulation of macrophage activation. Cell Mol Life Sci 2003; 60: 2334-46. 